\title{
Local strain energy based fatigue assessment of cruciform welded joints: experimental data analysis and influence of hot-dip galvanization
}

\author{
Luigi Mario Viespoli ${ }^{1, *}$, Francesco Mutignani ${ }^{2}$, Gabor Gulyas ${ }^{3}$, Heikki Remes ${ }^{4}$, and Filippo \\ Berto $^{5}$ \\ ${ }^{1-2-5}$ Department of Mechanical and Industrial Engineering, Norwegian University of Science and \\ Technology (NTNU), Norway \\ ${ }^{3-4}$ Department of Applied Mechanics, Aalto University, Finland
}

\begin{abstract}
Performing the fatigue assessment of a welded joint using the Notch Stress Intensity Factors [1] presents two major challenges. The first is the necessity of a precise reconstruction of the stress field around the notch tip, thus needing an extremely refined discretization with an evident computational cost. The other, is that the dimensions of the N-SIFs and so their critical values, vary accordingly to the William's solution [2] depending on the notch-opening angle. Consequence of this is that the mechanical properties necessary for the assessment vary as a function of the geometry treated. The research to overcome this issue has led to the use of the local Strain Energy Density [3]. The power of this parameter, used as a tool to perform fatigue assessment, consists of having a very low mesh refinement sensitivity [4], being the energy computed directly from nodal displacements and stiffness matrix [5], and having constant dimensions, so constant critical value for a given class of materials. In this paper, the local energetic method is applied to the analysis of the results of a series of tests performed on cruciform load carrying and non-load carrying specimens realized by S235 JRG2 structural steel plates. If load carrying, the fillet welded joints are made of $\mathrm{S} 355 \mathrm{~J} 2+\mathrm{N}$ structural steel. The fatigue testing has been performed in atmosphere at room temperature in as welded condition both with and without the corrosion protective zinc layer. Particularly, the interest is focused on the influence of the zinc layer of the fatigue properties of the joint and on the capability of the local energetic approach, confronted with the classic nominal stress approach, to accurately predict the fatigue failure. To conclude, the investigation of the tests executed reveals no significant difference in the fatigue life for the coated samples, compared with the uncoated specimens and the predictions according to the IIW recommendations [6].
\end{abstract}

${ }^{*}$ Corresponding author: “luigimv@stud.ntnu.no

\section{Introduction}

In many industrial applications the use of metals subject to corrosion, particularly in the case of steel and aluminum, presents the drawback of causing considerable expenses due to the maintenance or substitution of structures. The adoption of a surface treatment such as hotdip galvanization is a proven and diffused method to prevent the deterioration of the material. Is then important to understand the influence of the presence of the added layer of zinc on 
the mechanical properties of the base alloy, both in case of static loading and in case of fatigue, where the fracture initiation properties for a notched component can be heavily influenced. A reduction of the fatigue life with increasing zinc layer thickness was evidenced in tests performed on high-strength steel without stress concentration effect [7]. Other tests have, on the contrary, not found a systematic loss in fatigue life as a direct effect of the zinc layer thickness [8]. A wide study on the effect of the coating on the fatigue strength of ferritic steels shaped in un-notched geometries and, performing fatigue predictions on a KitagawaTakahashi diagram's based tool, it was found that the zinc layer does not affect the fatigue life up to a thickness of $60 \mu$ [9]. The influence of the coating on the fatigue life has been explored also for the cases of steel wires [10,11], different steel alloys with similar static characteristics [12]. All these results were obtained in the case of absence of geometrical stress intensifications, while fewer are present in the case of hot dip galvanized welded steel joints [13]. Further tests on different geometries and sizes are presented in this paper, investigating the influence of the zinc layer on the fatigue life of a welded joint and analyzing the results both in terms of nominal stress range and in terms of mean strain energy density range.

\subsection{Notes on the Strain Energy Density}

According to the volume based energetic criterion [3], failure occurs when the mean Strain Energy Density W, averaged over a control volume of radius $\mathrm{R}$ to be carefully identified and typical to the material, reaches a critical value $\mathrm{W}_{\mathrm{c}}$, which is not a property of the sharpness of the notch, as for the N-SIFs, but depends only on the material properties. This formulation has been then extended to non-zero radius notches and to the prediction of the crack initiation life. Considering valid the William's solution, the mean SED over an area $\Omega$ of radius $\mathrm{R}$ centered in the notch tip is:

$$
\bar{W}=\frac{e_{1}}{E}\left[\frac{K_{1}}{R^{1-\lambda_{1}}}\right]^{2}+\frac{e_{2}}{E}\left[\frac{K_{2}}{R^{1-\lambda_{2}}}\right]^{2}
$$

Where $\lambda_{1}$ and $\lambda_{2}$ are the William's eigenvalues, E the Young's modulus, $\mathrm{e}_{1}$ an $\mathrm{e}_{2}$ functions of the notch's opening angle and of the stress state. The N-SIFs are a function of the stress field, following the laws:

$$
\begin{aligned}
& K_{1}=\sqrt{2 \pi} \lim _{r \rightarrow 0}\left(\sigma_{\theta}\right)_{\theta=0} r^{1-\lambda_{1}} \\
& K_{2}=\sqrt{2 \pi} \lim _{r \rightarrow 0}\left(\tau_{r \theta}\right)_{\theta=0} r^{1-\lambda_{2}}
\end{aligned}
$$

For opening angles greater than $102^{\circ}$, the Mode II stress filed is non-singular and thus the dominant component in the singularity point is that of the Mode I stress field. The mean SED can so be approximated:

$$
\bar{W}=\frac{e_{1}}{E}\left[\frac{K_{1}}{R^{1-\lambda_{1}}}\right]^{2}
$$

In the hypothesis of validity of the Beltrami's failure criterion, $W_{c}=\Delta \sigma / 2 \mathrm{E}$, an estimate of the radius of the control volume is expressed as:

$$
R=\left(\frac{\sqrt{2 e_{1}} \Delta K_{1}}{\Delta \sigma}\right)^{\frac{1}{1-\lambda_{1}}}
$$

Important is to note that the formulation of the radius depends on the failure criterion adopted, which might be different according to which one suites better a particular load condition or 
a material. In practice, for each class of materials (i.e. welded structural steel) the radius is calibrated testing a multitude of different geometries, alloys and load ratios and seeking through iterations the critical radius which yields the minimum scatter. In the case of arc welded structural steel, the radius has been identified and validated as $\mathrm{R}=0.28 \mathrm{~mm}$, Fig. 1 .

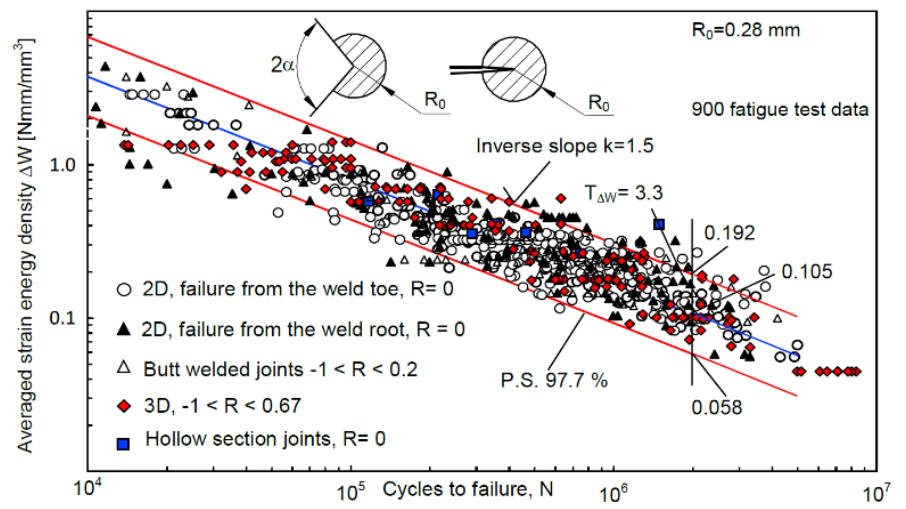

Figure 1. Fatigue band in local mean SED for arc-welded steel [4].

\section{Fatigue testing}

The joints tested consist of two different realizations of cruciform joint, one with load carrying fillet (Geometry 1) and one with non-load carrying fillet (Geometry 2). The specimens have been made of S235 JRG2 structural steel welded with automatic Metal Active Gas process. In case of load carrying fillets, these have been realized in S355J2+N structural steel. A part of the samples has been hot-dip galvanized to a zinc layer thickness in the range between 470 and $500 \mu \mathrm{m}$. No further thermal treatment has been executed and the testing has occurred in air at room temperature. Before the testing, all the samples were measured and grinded at the extremities in order to prevent any bending due to the rigid clamping in the testing machine.

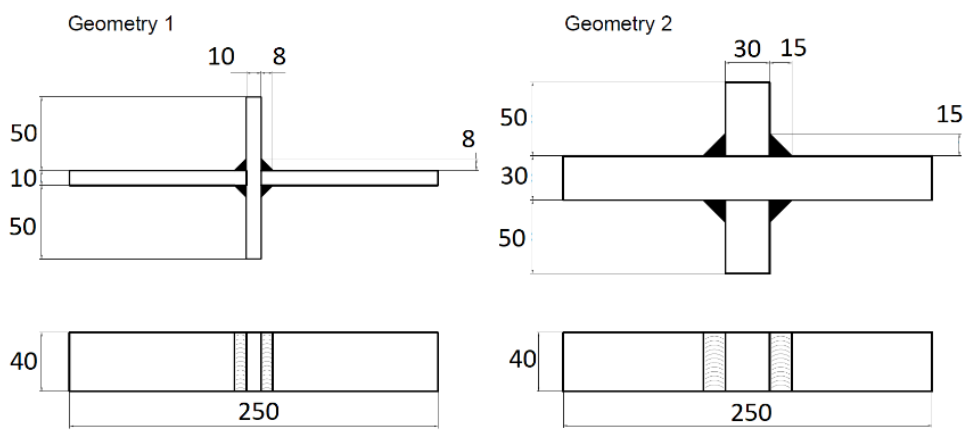

Figure 2. Geometries of the tested specimens.

The tests were performed on a servo hydraulic MTS 647 testing machine using cyclic axial tension loading with nominal load ratio $\mathrm{R}=0.01$. The frequency was set to $10 \mathrm{~Hz}$, lowered to $2 \mathrm{~Hz}$ when the displacement passed the value of $1.5 \mathrm{~mm}$ and the test continued until failure, when the total number of cycles was registered. The failure criterion adopted is the total tearing of the joint automatically detected by the testing system as a load drop.

\subsection{Test results}


A total of 35 Geometry 1, 30 of which hot-dip galvanized and 15 Geometry 2 joints were tested. The results are summarized in the following plots, showing the S-N bands extrapolated and the confront with the S-N curves suggested by the IIW recommendations [6]. The S-N curves for Geometry 2 have been corrected according to paragraph 3.1. All the failures occurred, as typical for these details, at the weld toe or root. At last, the data have been reanalyzed in terms of mean SED, and all of them fit in the energetic fatigue band for welded structural steel details already available in the literature, regardless of coating, geometry or size.
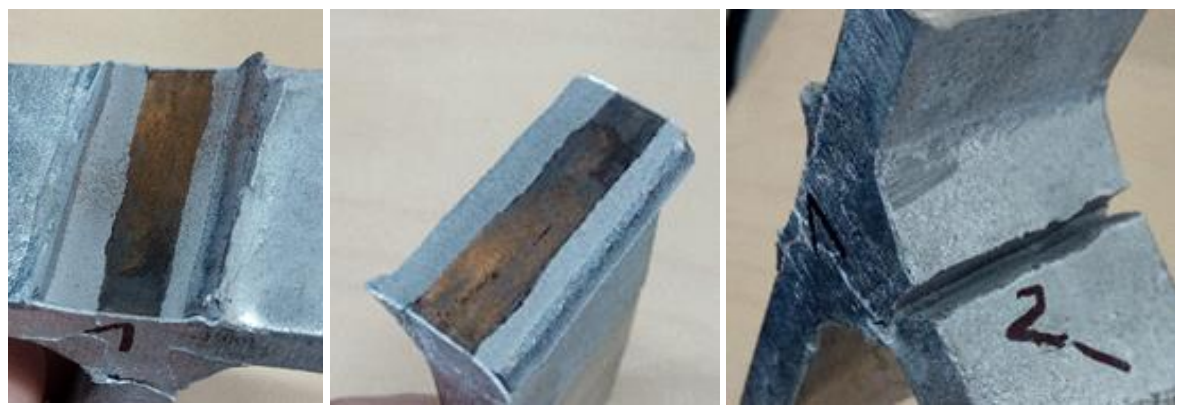

Figure 3. This specimen is an example of both the failure mechanism for the tested detail, Geometry 1: it failed for initiation at the weld root with propagation through the weld (left and center), while, contemporarily, a second crack initiated and propagated at the weld toe on the other side (right).

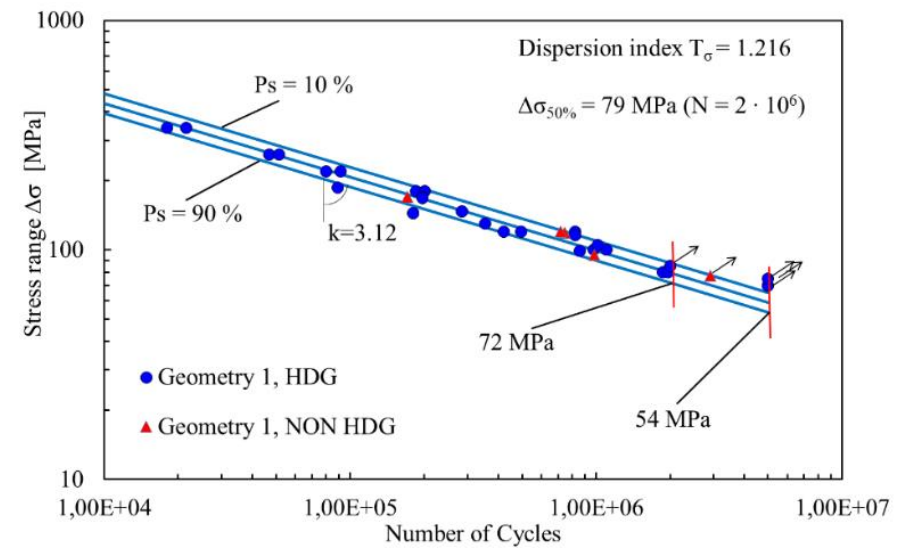

Figure 4. Results and fatigue band for Geometry 1 in terms of nominal stress range, both coated and uncoated.

While the fatigue testing has been executed for a nominal load ratio of $\mathrm{R}=0.01$, the fatigue curves reported by IIW give the nominal strain range for each class of joint in the case of nominal load ratio $\mathrm{R}=0.5$. IIW suggests, in order to account for the residual stresses, not relieved by any treatment after the welding process, to not correct the strain range for $\mathrm{R}<0.5$ in case of thick walled components and in presence of residual stresses. The effect of the thickness is evaluated according to paragraph 3.1. 


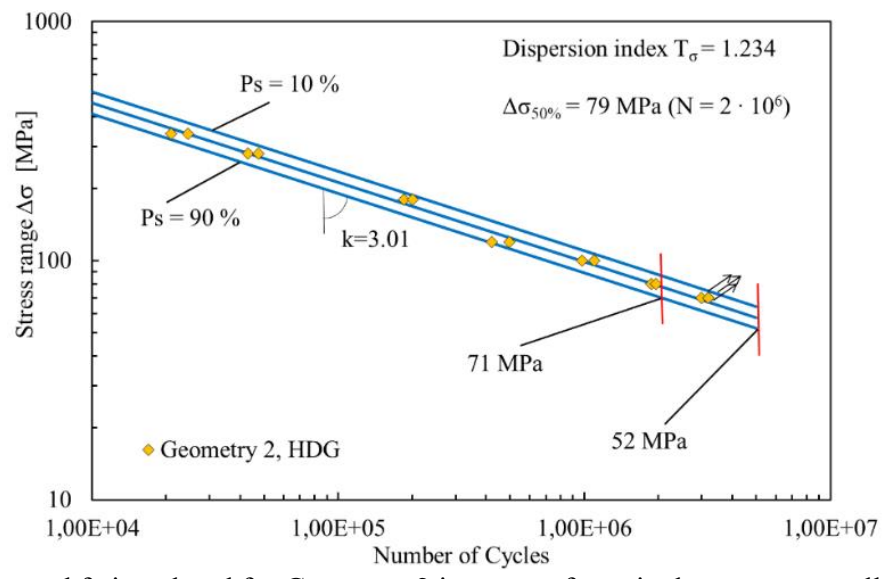

Figure 5. Results and fatigue band for Geometry 2 in terms of nominal stress range, all the samples are hot dip galvanized.

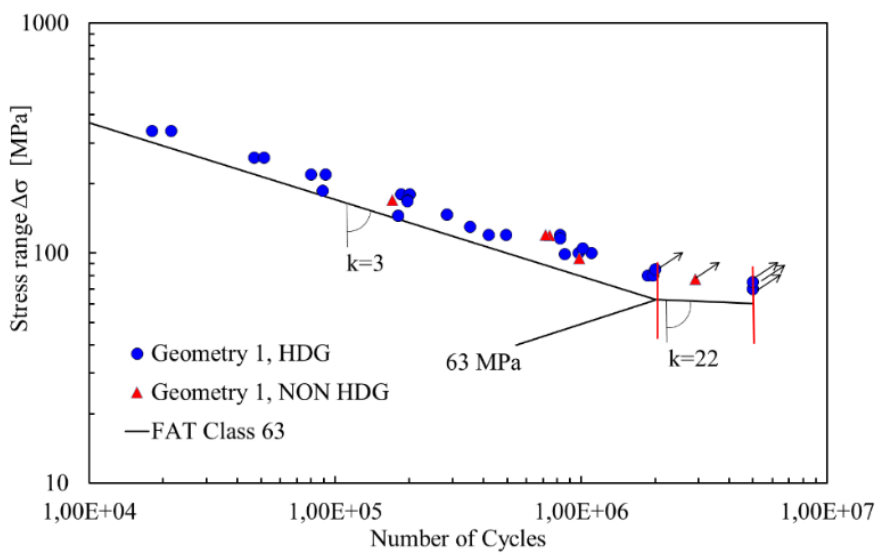

Figure 6. Testing results for Geometry 1 in terms of nominal stress range. The samples are compared with the relevant fatigue class (FAT 63) according to the IIW recommendations [6].

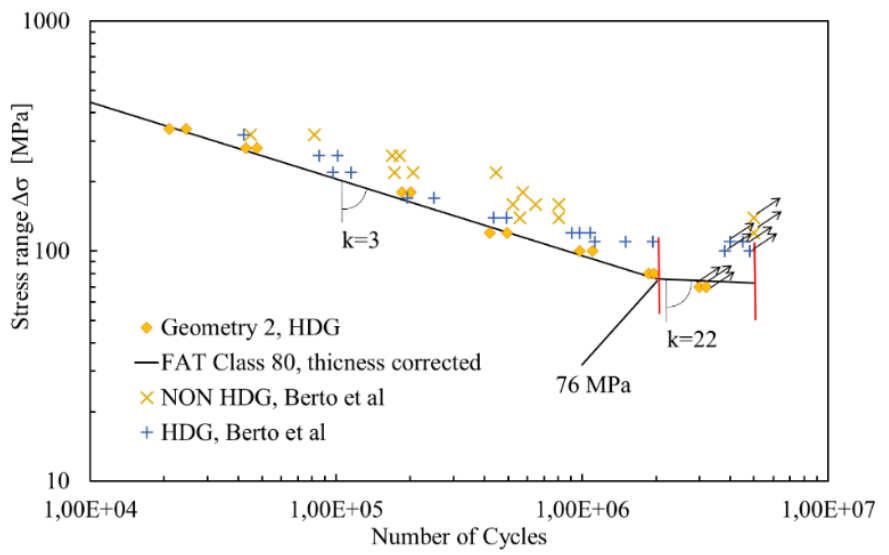

Figure 7. Testing results for Geometry 2 in terms of nominal stress range. The samples are compared with the relevant fatigue class (FAT 80) according to the IIW recommendations [6]. It is evident how the HDG Geometry 2 samples do not match the resistance of the thinner samples tested by Berto et al [13] (see 3.2). 


\subsection{Results according to local energetic analysis}

The fatigue data have been reanalyzed in function of the mean Strain Energy Density, to verify whether the coated joints fit into the fatigue energetic band proposed for welded structural steels. In order to extract the mean SED over the control volume (surface in a 2D analysis) of radius $\mathrm{R}$, it is necessary to create a FEM model of the joints, represented by an ideal isotropic linear elastic continuum in the nominal dimensions. This means neglecting the imperfections which are present both as deviation from the nominal dimensions and as variability of the material properties from the ideal due to the welding process. All these errors are already considered into the material properties, in order to simplify the assessment. In particular, a quarter of the joint can be modeled with a coarse mesh of planar quadratic elements in plain strain condition, imposing double symmetry boundary. A circular area of radius $\mathrm{R}$ is introduced at the critical point, the weld root for Geometry 1 and the weld toe for Geometry 2, on which the mean SED is computed.

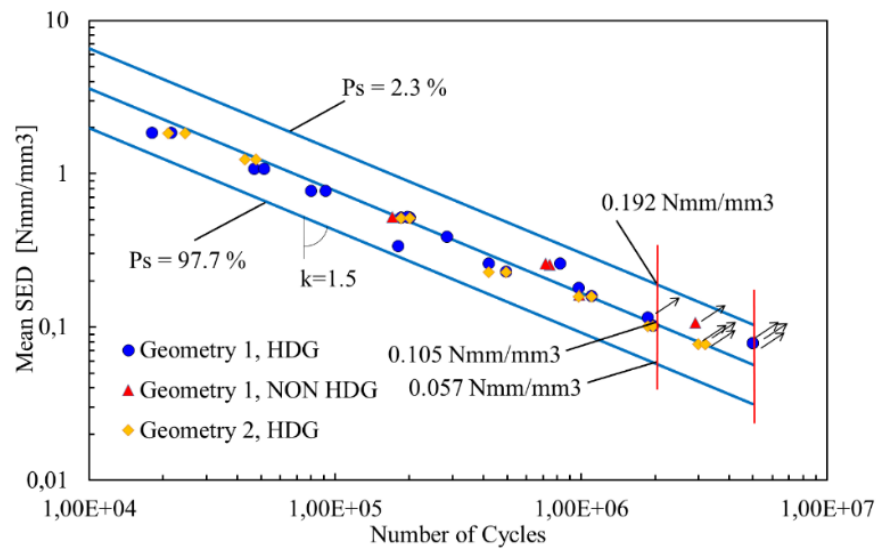

Figure 8. Test results in terms of mean SED range.

The results obtained show how the samples tested fall perfectly into the fatigue band for arcwelded structural steels, with no evident decrease in fatigue life due to the hot-dip galvanization process. The method, although requiring the use of a FEM tool, overcomes the uncertainties on the actual stress field typical of the much simpler Nominal Stress approach, as described in the following paragraph.

\section{The dimension effect}

\subsection{For Nominal Stress predictions}

According to the recommendations in the IIW document, the thickness of the plates is to be considered in case of crack initiating at the weld toe, for effective thickness greater than 25 $\mathrm{mm}$, which is the reference of the curves provided. The thickness effect is evident confronting the fatigue resistance of the samples Geometry 2 with an analogous non-load carrying geometry realized with plates of $10 \mathrm{~mm}$ of thickness [13] instead of $30 \mathrm{~mm}$ as for the tests presented in this paper (Figure 6). In order to account to the loss of strength with increasing thickness for Geometry 2, IIW suggests the following exponential relation:

$$
f(t)=\left(\frac{t_{r e f}}{t_{e f f}}\right)^{n}=\left(\frac{25}{30}\right)^{0.3}=0.9468
$$


Where $n=0.3$ is the exponent for cruciform joints. The value of the FAT class is then diminished by multiplying for the factor.

\subsection{SED thickness analysis}

This effect can also be easily accounted using the mean SED as parameter for the fatigue assessment and considering the scale effect for the N-SIFs [1]. Considering two geometrically similar joints "a" and " $b$ ", of characteristic lengths $t_{a}$ and $t_{b}$, being the index "i" 1 or 2 according to the loading mode and $\lambda_{\mathrm{i}}$ the relevant William's eigenvalue, the correlation between the N-SIFs of the two joints is:

$$
K_{i, b}=K_{i, a}\left(\frac{t_{b}}{t_{a}}\right)^{1-\lambda_{i}}
$$

Recalling that the mean SED can be expressed in terms of the N-SIFs and other geometrical parameters as:

$$
\Delta \bar{W}=\frac{1}{E} e_{1} K_{1}^{2} R^{2\left(\lambda_{1}-1\right)}+\frac{1}{E} e_{2} K_{2}^{2} R^{2\left(\lambda_{2}-1\right)}
$$

And that for an opening angle superior to $102^{\circ}$ the, as in the case of the geometry 2 , in which the failure occurs at the weld toe with an opening angle of $135^{\circ}$, the Mode II stress field is non-singular and can thus be neglected, the mean SED for the two geometrically similar joints can be expressed as:

$$
\left\{\begin{array}{l}
\Delta \bar{W}_{a}=\frac{1}{E} e_{1} K_{1, a}^{2} R^{2\left(\lambda_{1}-1\right)} \\
\Delta \bar{W}_{b}=\frac{1}{E} e_{1} K_{1, b}^{2} R^{2\left(\lambda_{1}-1\right)}
\end{array}\right.
$$

After few simple passages and considering the size of the Geometry 2 presented in this paper and the joints presented in [13]:

$$
\Delta \bar{W}_{b}=\Delta \bar{W}_{a}\left(\frac{t_{b}}{t_{a}}\right)^{2\left(1-\lambda_{1}\right)}=\Delta \bar{W}_{a}\left(\frac{30}{10}\right)^{2(1-0.674)}=\Delta \bar{W}_{a} \cdot 2.05
$$

This explains how two similar geometries of different size (the Geometry 2 here presented and the non-load carrying geometry in [13]), show a different fatigue life for the same nominal strain range. In order to double check, considering a nominal stress of $180 \mathrm{MPa}$ for both joints, the FEM analysis provides a mean SED over the critical volume of radius $\mathrm{R}=0.28$ $\mathrm{mm}$ around the weld toe equal to 0.2728 and $0.5125 \mathrm{Nmm} / \mathrm{mm}^{3}$ for the joints made from plates 10 and $30 \mathrm{~mm}$ thick respectively. The ratio between the values is 1.88 , in good agreement with the analytical approximation presented.

\section{Conclusions}

The fatigue strength of hot-dip galvanized and uncoated load carrying and non-load carrying cruciform MAG welded structural steel joints has been tested to understand the influence of the zinc layer on the fatigue life of a welded detail in presence of stress intensification. The resistance shown by Geometry 2 samples evidences how the thickness of the plates forming the joint is an important parameter to be taken into account in the assessment, even though this might, for certain details, be not reported in manuals for the fatigue resistance evaluation with the nominal stress. The use of the mean Strain Energy Density for the fatigue assessment allows to overcome this problem and to provide a valid estimate for every geometry, 
dimension and opening angle. The use of the energetic method could be simplified in order to skip the FEM analysis every time a detail is to be verified for fatigue life creating a database of mean SED for each detail for unit stress and for a set arbitrary dimension. The value of energy can then be computed easily recalling that the ratio of the SED for two identical components is equal to the square of the ratio between the nominal stresses and recalling the correlation between the SED for two proportionally scaled components. Although the results of the particular tests presented in this work show that there is no significant negative influence of the coating on the fatigue performance of the tested details, it should be pointed out that the geometry tested both in coated and uncoated conditions is the load-carrying, in which the weak point is the crack due to the not complete penetration of the weld and where the coating is not present. On the contrary, in a precedent work on a similar, but non-load carrying geometry, a slight reduction in the fatigue properties had occurred due to the presence of the zinc layer. The conclusion is then that this reduction is present and should be considered if the affected zone is already the weak spot for the analyzed detail, while is not strong enough to alter the failure dynamics if the catastrophic crack is typically initiated in a point where the coating is not present.

\section{References}

1. P. Lazzarin, R. Tovo. Fatigue \& Fracture of Engineering Materials \& Structures. 21, 10891103 (1998)

2. M.L. Williams. Journal of Applied Mechanics. 19, 526-528 (1952)

3. P. Lazzarin, R. Zambardi. International Journal of Fracture 112, 275-298 (2001)

4. P. Lazzarin, F. Berto, M. Zappalorto. International Journal of Fatigue. 32, 1559-1567 (2010)

5. O.C. Zienkiewicz, R.L. Taylor, J.Z. Zhu. Sixth Edition. Elsevier Butterworth-Heinemann. (2005)

6. A. Hobbacher. IIW collection, Springer. (2016)

7. Y. Bergengren, A. Melander. International Journal of Fatigue. 14(3), 154-162 (1992)

8. R.S. Browne, N. Gregory, S. Harper. In T. Organization (Ed.), Proceedings of a Seminar on Galvanizing of Silicon-Containing Steels. Liege, Belgium: ILZRO Publishers. 246-264 (1975)

9. J. B. Vogt, O. Boussac, J. Foct. Fatigue and Fracture of Engineering Materials and Structures. 24(1), 33-39 (2001)

10. J.H. Jiang, A.B. Ma, W.F. Weng, G.H. Fu, Y.F. Zhang, G.G. Liu, G.M. Lu. Fatigue and Fracture of Engineering Materials and Structures. 32(9), 769-779 (2009)

11. W.J. Yang, P. Yang, X.M. Li, W.L. Feng. Materials and Corrosion, 63(5), 401-407 (2012)

12. K. Berchem, M.G. Hocking. Materials Characterization, 58(7), 593-602 (2007)

13. F. Berto, F. Mutignani, L. Pittarello. Procedia Structural Integrity. 2, 1813-1820 (2016) 